\title{
Chemical and Physical Properties of Dye-Stuffs*
}

\author{
Acid and Basic Dyes in Gelatin Solutions
}

\author{
By J. Traube and F. Koehler
}

[The original article is both too long and somewiat too technical to be reproduced here in its entirety. It is of such great value, however, especially in view of the remarkable expansion of our dyeing industry that we are glad to publish an abstract of such portions of it as are of general interest.The Editor.]

$\mathrm{I}$ $\mathbf{N}$ a previous number of this journal (1915, II, 42) we published an account of our researches concerning a large number of salts, acids, bases, nareotics, etc., with respect to the degree of rapidity with which a gelatine jelly at a given temperature (about $26^{\circ} \mathrm{C}$.) passes over into the hydrosol conditions upon the addition of one of the said substances, as also the rapidity of formation of the gelatine jelly from the hydrosol condition in the presence of the acid substance. We found ourselves in agreement with the previous researches of Pauli, Schibig an others, to the effect that the dissolving of gelatine corresponds to a process of swelling while the formation of jelly corresponds to a process of shrinking. We found further that the laws determined for gelatine are applicable to other colloids such as albumen. This suggested that it would be of especial interest to extend these researches to the domain of dyestuffs. . . .

We are indebte to the "Badischen anilin-und Sodafabrik" for the following dyes, rhodamin $\mathrm{B}$ extra, night blue, rose aniline, eosin B extra, erythrosin G, pure red B conc., phloxin P, Bengal rose A.T., anthraquinon-green G.X.N., wool-violet $\mathrm{S}$, methyl-green, crystal-violet, methylene-green BX, Toluidinblue, methylene-blue, Nile-blue A conc., malachite-green BX, indigo-carmine.

From L. Casella \& Company we received new-blue R, cyanolpure-green $\mathrm{G}$, water-blue, acid-green extra, tannin-heliotrope, cyanol-green 6G, formyl-viølet SB, brilliant walk-blue, indazin $\mathbf{N}$, acid-violet $\mathrm{BS}$, naphtindon $\mathrm{BB}$, isamine-blue $6 \mathrm{~B}$, tetracyanol SF, diamond phosphine $\mathrm{R}$, cyanol FF, trypan-red and patent-blue $\mathrm{V}$.

From C. A. F. Kahlbaum we obtained safranin G extra, Bismarck-brown extra, neutral-re extra, quinolin-yellow, martiusyellow, picrin-acid, gentian-violet $\mathrm{BR}$, heliotrope $\mathrm{BB}$, pure brown G, Congo-red, orange I, napthol-green B, azo-blue, azoruby $\mathrm{S}$, Bordeaux $\mathrm{R}$, naphtol-yellow $\mathrm{S}$, thionin-blue, methylorange, brilliant-Congo $\mathrm{G}$, light-green $\mathrm{S}$, diamine-blue $3 \mathrm{~B}$, guinea-green $\mathrm{B}$, Victoria-blue $\mathrm{B}$, and rhodamin $\mathrm{B}$.

Finally, we obtaine from still other sources benzo-purpurin $\mathbf{B}$ and alizarin-saphiøl, chrysaniline $\mathbf{S}$, and chrysoidin $\mathbf{S}$.

It is only too well known that in the case of many of these dyestuffs one must take into account certain impurities and adulterations.

THE RAPIDITY OF SOLUTION AND OF FORALA TiON OF A GELATINEI JELLY.

We made use here of the same simple method employed in our previous work: $6 \mathrm{ccm}$. of a 0.1 per cent dyestuff solution was mixed with $4 \mathrm{ccm}$. of 6 per cent perfectly pure and neutrally reacting Nelson's gelatine which had shortly before been heate to about $50^{\circ} \mathrm{C}$., regeant glasses of equal with being employed. After a certain lapse of time the glasses containing the congeale gelatine were place in a thermostat (usually at $26^{\circ} \mathrm{C}$.) and the melting of the gelatine was determined by means of small balls made of solid glass (these being better than beals). In the control solutions water was added in place of the dyestuff solution. The direct error of observation could not have been greater as a general thing than from one-half minute to one minute; however, in jellies

*Translated for the Scientific American Monthly from the Intern tionale Zeitschrift fuer Physikalisch-Chemische Biologie (Berlin). of almost complete opacity, which were illuminated with an arc light, the error may be somewhat greater.

Since it was observed that the time required for solution of the jelly apart from the substance added depended primarily upon the length of time it took the jelly to form, the solutionperiods for pure gelatine and for various dye-stuff gelatines were first determined... The jellies were kept in an ice box. [Table omitted.] The first observation made was that the well-known continuous alteration (lasting for weeks) of a gelatine jelly, which can be perceived by the lessening of the vapor pressure, etc., corresponding to a shrinking process . . . and since such a hysteresis is not confine to gelatine alone this gradual process of shrinking is of much significance with respect to manifold biological and technical questions concerning the most various colloids, problems for example concerning dyeing and tanning processes, the period of incubation, etc.

It was further observed that the highly colloidal basic dyestuff, night blue (which contains dextrin) has a strong shrinking effect while the weakly basic rhodamin $B$ behaves not much unlike water. Among the acid dyestuffs anthraquinon, like benzo-purpurin, has a strongly swelling effect while Bengal rose which like night blue does not permeate plant tissues-after a lapse of three days-has a shrinking effect. ...

We next allowed the gelatine solutions which had served for the experiments to congeal once more and after a lapse of twenty-four hours place them again in the thermostat at $26^{\circ} \mathrm{C}$. and noted the time require for solution. [Table omitted.]

Later, the gelatines were again place in the thermostat (after a lapse of another twenty-four hours) after previously being congeale in a freezing mixture at $-10^{\circ} \mathrm{C}$. and the solution time again noted. [Table omitted.]

It was found that after several days dye-stuif jellies, such as the gelatines of anthraquinon benzo-purpurin in which the dye-stuffs have a swelling action gradually undergo liquefaction starting from the surface even at ordinary temperatures or in the ice box, while in the case of dye-stuffs which exert a shrinking action, such as Bengal rose, night blue, etc., a similar liquefaction was not observed even after eight days. This phenomenon which was discovere to be quite general among the various dyestuff jellies examined is partly, but not entirely, dependent upon the collecting of bacteria and bacterial toxins.

It must be noted that the above mentioned dye-stuff-gelatine hydrosols, also can be refrigerate at $-100^{\circ} \mathrm{C}$. into solid carbon dioxide plus ether. The subsequent melting at $26^{\circ} \mathrm{C}$. revealed, however, only very slight differences in the solution times compared to hydrosols which had been congealed at $0^{\circ} \mathrm{C}$. . . .

Since the differences in the degree of shrinking and swelling of the dye-stuff jellies increase in proportion with the duration of the time during which the jelly is kept before the solution test, the following experiments were all carried out in such a manner that the jelly had previously been kept seventy-two hours in the jce box. The degree of concentration of the jellies was the same as above and the temperature in the thermostat was $26^{\circ} \mathrm{C}$. to $26.5^{\circ} \mathrm{C}$. Several days later (four to eight days) the experiment was repeated at a temperature of about $28^{\circ} \mathrm{C}$. [Here follow the series of figures obtained which are omitted.]

The most noteworthy result of our experiments as shown in the tables is the demonstration that basic dyestuffs exert a shrinking action while acid dye-stuffs, on the other hand, exert chiefly a swelling effect, though a lesser portion of them 
also have a shrinking effect. The acid dye-stuffs which exert a shrinking action (Bengal rose, erythrosin, phloxin, pure red, pure brown, azo blue, etc.) belong as we shall see to the impermeable or slightly permeable dye-stuffs. It is these whose jellies do not deliquesce in the air.

It is a well-known fact that the non-crystallized basic dyestuffs sometimes contain admixtures of dextrin, etc., while the acid dye-stuffs contain sodium sulphate, sodium bisulphate, etc. Since, however, only 0.06 per cent of the dye-stuff was contained in the jelly, the small quantities of such salts present are practically without influence upon the swelling; other experiments proved that even 0.5 per cent of dextrin had no influence upon the swelling. ...

In the shrinking and swelling action exerted by colloids on the part of dye-stuffs we believe we have discovered a new factor which sheds light upon the most various phenomena not only in the technology of dyeing and tanning but in histology and possibly in photography, since what has here been proved true with regard to gelatine is likewise applicable to numerous other colloids, albumen, the fibers of plants and animals, etc., as shown by our experiments.

The greater the shrinking effect exerted by a dye-stuff the less necessary is it in general to assist the fixation by the employment of a special mordant. [The accompanying tables are here omitted.]

As shown by the tables basic dye-stuff may be expected as a usual thing to accelerate the formation of the jelly while most acid dye-stuffs retard the formation.

THE CAPACITY FOR DIFFUSION IN JELLIES AND ITS RAPIDITY; ALSO SWELLING AND SHRINKING IN RELATION TO CELL PERMEABILITY.

In a comprehensive study concerning the absorption of colloids by the vegetable "plasma-skin" Ruhland arrives at the conclusion that the capacity for diffusion and the rapidity of diffusion of dye-stuff solutions in jellies, especially in gelatine jelly, run parallel "without exception" to the capacity of dyestuffs to penetrate living plant cells; also that "the living cell resembles an ultra-filter operating at a high pressure by regson of its semi-permeable plasma-skin with respect to colloids," and likewise that "the dispersive power of the jellies of the plasma-skin constitutes the decisive moment for the capacity and rapidity of absorption."

Ruhland's views are highly esteemed and justly so, and we should probably have never thought of criticising them but for the circumstance that the facts proved by us with regard to the capacity for shrinking of the basic dye-stuffs and the capacity for swelling of numerous acid dye-stuffs have forced upon us the conclusion that the capacity for swelling and for shrinking and therewith the capacity likewise of jellies to liquefy, or to congeal, as well as to lessen or to increase the friction of the cell sap, must be one of the factors which exerts an influence upon the permeability, and especially too upon the "storage" and rapidity of storage of dye-stuffs.

[We are obliged here to onit the detailed criticism made by the authors concerning Ruhland's methods and conclusions together with the elaborate tables which accompany it, merely giving their final conclusions.-Enitor.]

When we compare the permeabilities determined by Ruhland (which must be determined for basic dye-stuffs in other plant objects as well as for acid dye-stuffs) with the diffusion values obtained by me, we undoubtedly perceive that they largely coincide. In spite of this it is not possible to regard the penetration of plant cells by dye-stuffs merely as a diffusion in jelly-in other words as an ultra-filtration.

Among the basic dye-stuffs we will refer only to rhodamin B and Bismarck brown; it is well known that in many dyestuffs the differences of diffusion are very great where gradations of permeability are not specified.

With respect to acid dye-stuffs we found very marked exceptions in the case of wool-violet and Martius-yellow. We may mention, also, methyl-orange and fast-brown $G$ which diffuse readily but permeate with considerable difficulty. Eosin and phloxin and also erythrosin and Bengal-rose diffuse better than might be expected from their very low degree of permeability. Acid green, light-green $\mathbf{S}$, and naphtol-green permeate readily and diffuse poorly.

The very circumstance that we found very different permeability values for basic and acid dye-stuffs (though making use of similar sections of plants) whereas the figures for the jelly diffusion of acid and basic dye-stuffs exhibit similar intervals, indicates that there are concerned impermeability influences other than jelly diffusion alone. We know that salt ions such as potassium, ammonium, etc., penetrate cells with comparative ease because of the fact that they have swelling properties (just as narcotics do) ; but we also know, on the other hand, that shrinking ions such as calcium, $\mathrm{SO}_{4}$, or nonconductors such as varieties of sugar penetrate only with great difficulty. When we find, therefore, that basic dyestuffs nearly always have a shrinking action, while on the other hand acid dye-stuffs mostly, or at least very frequently (and in increasing measure with the lapse of time) exert a swelling action, we can hardly avoid the conclusion that we have here the key to the different behavior of basic and acid dye-stuffs with respect to capacity for storage and rapidity of storage, and furthermore, that the properties of swelling and shrinking cannot be excluded from the problem of permeability.... We are of opinion that when dye-stuffs such as eosin, phloxin, erythrosin, and Bengal rose permeate less readily than they diffuse in jellies, it is necessary to take into consideration the shrinking properties of these dye-stuffs (these properties being greatest in the poorly permeating dye-stuff Bengal-rose).

JELLY DIFFUSION AND DIALYSIS.

[This section begins with a table here omitted, giving the rapidities of jelly diffusion and dialysis as noted by various observers.-EDITOR.]

An examination of the foregoing table justifies us in concluding that the rapidity of gelatine diff usion is parallel in general to rapidity of dialysis. Only in the case of neutral-red is there a slight difference. Furthermore, the dialysis values ... are much less exact than the values of the jelly diffusion and are derive moreover, from various observers. According to Hoeber, guinea-green $\mathrm{B}$ dialyses rapidly, while according to our own observation guinea-green $\mathrm{R}$ diffuses very slightly in gelatine. The value concerned is $4(2)$.

According to Teague and Buxton dye-stuffs are arranged with respect to the capacity for diffusion in one per cent agar almost in the same sequence as in respect to their capacity for dialysis through a cellulose filter. Eosin, methylene blue, chrysoidin, and safranin diffuse strongly, Congo-red and azureblue diffuse very slightly, and night-blue not at all.

While we by no means claim that the rapidity of diffusion and that of dialysis run parallel to each other without exception, the coincidence of values shown above leads us to conclude that the permeability of plant cells may be regarded quite as justifiably as a simple dialytic process, as it can be considere as a process of jelly filtration. We do not deny, indeed, the possibility that jelly filtration occurs in plants, but convincing proof thereof is thus far not at hand.

The analogy of the results given by gelatine, agar, and cellulose, justifies us in believing that for different colloids the same or nearly the same sequence of diffusing and dialyzing substances exists.

THE COLLOIDAL CONDITION OF DYE-STUFFs.

Ruhland has justly observed that the direct ultra-microscopic examination of dye-stuffs has laid bare many contradictions. Since dye-stuffs are frequently impure, containing small admixtures of sodium-sulphate, dextrin, etc., which influence the ultra-microscopic image, such contradictory results are not to be wondered at. In spite of this we are of opinion that more precise ultra-microscopic investigations are best adapted to furnish a concept of the colloidal condition of various dyestuff substances.

Ruhland believes that certain indirect methods, like the 
method first proposed by Hoeber of the precipitability of acid dye-stuff solution by varying concentrations of calcium chloride or nickel-chloride, as likewise the jelly diffusion methods of Bechold (utilizing the principle of ultra-filtration) are to be preferred, although these methods are certainly far from perfect.

Pelet-Jolivet correctly points out that the methods of precipitation are primarily related to a chemical exchange. The sodium salt of the dye-stuff is exchanged at least partially with the calcium salt or nickel salt concerned, whose difficult solubility will primarily influence the process. In spite of this we are also of the opinion that in general, or at any rate usually, the values obtaine by this method admit of the conclusion as to the dispersive power, but the method should be controlle by the direct ultra-microscopic method.

The same restrictions must be applied to the principle of ultra-filtration.

If this principle be strictly accepted the measurements of diffusion would yield an exart neasure of the dispersive power and if we admit further trat the rapidity of diffusion and permeability always run parallel, we shall arrive at the theory advocated by Ruhland according to which the magnitude of the colloid particles is decisive with respect to the permeability. But this is really a revival of the old "sieve theory" of M. Traube-at any rate so far as colloids are concerned.

Attention must be called, however,-in opposition to the principle of ultra-filtration-to the alterability of the dispersing jelly through the substance added. If we filter through gelatine various disperse particles of the same colloid or different colloids having the same degree of swelling or shrinking effect, we are justifie in determining in this manner the relative magnitude of the particles, but if we try to compare dye-stuffs having a marke swelling effect with those having a marke shrinking effect, we shall inevitably arrive at false conclusions concerning the degrees of dispersive power. Thus for example, a dye-stuff of the eosin group may be only slightly colloidal, and yet in consequence of its capacity for shrinking it may take the place of a highly colloidal condition. In spite of these restrictions, however, which must be considere in estimating the value of the ultra-filtration method, we are of opinion that it is usually possible to obtain in this manner an approximately correct idea of the dispersive porwer of the dye-stuff colloid. However, Ruhland's "sieve theory" should be restricted in this case also by saying that the diffusion and permeability are influenced, not only by the magnitude of the particles but also by the capacity for swelling or shrinking of the dispersoid with respect to the dispersion medium.

MOLECULAR WEIGHT, COLLOIDAL CONDITION AND DIFFUSION, ETC.

In a work by Traube and Onodera it was shown that in aqueous solutions of free alkaloids sub-microns not become visible until the molecular weight exceeds a certain minimum magnitude. Thus there is indicated a dependence of the colloidal condition upon the molecular weight; however it is pointe out that the molecular weight is not the only factor in question, but that constitutional influences are present, though, perhaps, of secondary character. It was not known at that time to one of us-namely Traube-that similar views hal already been propose with respert to dye-stuffs. Krafft has pointed out the significance of the molecular weight with respect to the colloidal properties of dye-stuffs. Buxton and Teague have shown furthermore that the capacity for dialysis and therewith the dispersive power, in spite of certain deviations, bear a quite unmistakable relation to the molecular weight; Wo. Ostwald has published in his volume of Colloidal Chemistry a table in which the same results are indicated.

[Here follows a table which we omit showing the degree of parallelism between molecular weights and colloidal condition. -Editor.]

To begin with we would like to call special attention to the facr that the jelly diffusion can by no means be regarded always as a measure of the dispersive power. We may also remark that the high molecular weight of the group: Eosin, phloxin, erythrosin, and Bengal rose, can be traced to the greater number of atoms of chlorine, bromine, or iodine; , furthermore, the nature of the atom doubtless plays a part. With the exception of these dye-stuffs, however, it cannot be doubted that in general the capacity to form colloidal aggregates-as in the alkaloids-increases in proportion with the molecular weight, even though other influences cannot be excluded. This relationship between molecular weight and dispersive power is of the highest importance with respect to the principles of colloidal chemistry. ...

\section{CONCERNING STORAGE AND DYEING.}

.. . The rapidity of storage is an exceedingly important factor. We will now enquire what factors are chiefly operative in causing a dye-stuff to be precipitated at a definite locus of the cells.

The reason that the basic dye-stuffs have a considerably greater capacity for storage than the majority of the acid dye-stuffs, is to be attribute above all to their property of producing a shrinking effect. The greater the shrinking effect exerted by the substance the less will be its need of a mordant in order to fix it. Dye-stuffis which have a swelling effect exhibit a tendency to permeate while those which have a shrinking effect have a tendency, on the contrary, to allow themselves to be absorbed. A dye-stuff having a strong shrinking effect, such as night-blue or naphtindon, naturally exhibits this tendency in a far greater degree than a slightly shrinking dye-stuff of great dispersive power, like methyleneblue, etc. Krafft has already pointe out that it is also true for highly. colloidal acid dye-stuffs, like azo-blue and diaminpure blue that they are capable of dyeing cotton without a mordant.

In our diffusion experiments in which $5 \mathrm{ccm}$. of 10 per cent gelatine were place in small test tubes and covered with 1 $\mathrm{ccm}$. of a 0.1 per cent solution of dye-stuffs, we found that after a lapse of eight days a complete clarification of the dyestuff solution occurred in the aqueous superficial solution of azo-blue, isamin-blue, naphtindon, and new-blue, with a deposit of the dye-stuff upon the surface of the gelatine. We are here concerned with highly colloidal dye-stuffs which, like azo-blue, isamin-blue, and naphtindon, undergo no diffusion in the jelly, or which like new-blue diffuse very slightly.

An almost complete clarification with a dyeing of the gelatine surface was observe in the case of heliotrope, waterblue, fast-brown, Congo-red, fast-red, Nile-blue, Bordeaux, Victoria-blue, brilliant-Congo, and alizerin-saphiol. These are all highly colloidal dye-stuffs and the process of dyeing can be most clearly observed here. The more dispersive dye-stuffs, which diffuse more strongly in the gelatine not exhibit this capacity for storage or else show it only in a slighter degree; furthermore it need scarcely be mentioned that in the absence of the gelatine phase even the highly colloidal dye-stuffs are not deposited. The gelatine surface exerts an attraction upon the particles of dye-stuff whether these be acid or basic, provide only the said particles are of sufficient magnitude. The particles become aggregated and form upon the surface of the jelly a cohesive layer of the dye-stuff of measurable thickness. But it must be emphasized that we are here concerned with a colloidal and not a chemical process, as is true in general of dyeing processes.

It is a known fact that the capacity for absorption of substances frequently bears a close relation to the activity of the surface. In the section following are containe the superficial activities of the dye-stuff solutions here investigated. It might be thought that a dye-stuff of great surface activity, e.g. rhodamin $B$ would be absorbe in greater measure, in spite of its great dispersive power, than methylene-blue for instance; however, basicity also plays a part here and we lack technical information concerning the dyeing power of the various dye-stuffs as such, to enable us to express this relation between surface activity and storage capacity. 
We are concerned naturally in the consideration of the dyeing capacity and storage capacity of dye-stuffs, not merely with the dye-stuff itself, but above all with the electric charge of the locus in which the storage takes place, with the presence of the substances which are found there, with the medium in which the dye-stuff operates, and particularly with the possible addition of a third substance to the medium involved.

We must, therefore, at once reject the hypothesis of those authorities who uphold the chemical theory of the process of dyeing according to which the fibers must contain basic or acid groups in order to absorb acid or basic dye-stuffs respecitvely. It is certainly true that when at the loci in question an-ionic colloids (like tannin) are found dye-stuff cations are there deposited and vice versa; but on the other hand the presence of free hydrogen ions at once accelerates the deposit of dye-stuff am-ions and on the other hand the presence of hydroxul ions accelerates that of dye-stuff cat-ions. It is well known in fact that it it customary in the technology of dyeing to add to the sodium salts of the acid dye-stuffs, assistant acids such as sulphuric acid, etc., or acid salts, while to basic dye-stuffs sodium carbonate, etc., are sometimes added.

[We here omit a series of detailed experiments along this line, giving merely the definite conclusions of the authors.EDITOR.]

Taking everything into consideration we must again lay stress upon the fact that in our opinion the storage and dyeing process is colloidal in character and not stoichiometrical-chemical in spite of the partial decomposition of the dye-stuff salt. Definite influences are exerted by the nature of the dye-stuff, the respective acidity or alkalinity of the dye-stuff ion, the swelling or shrinking properties connected therewith, the dispersive power of the.dye-stuff and apparently, also, the surface activity. A definite influence is exerted furthermore by the acid or basic reaction of the medium and the storage locus (limiting surface of the phase) as, likewise, the presence of colloids having opposite electric charges, which occasions a flocculent separation. All the influences which occasion a lessening of the dispersive power of the dye-stuff increase the absorptive power.

\section{THE SURFACE TENSION OF DYE-STUFF SOLUTIONS.}

[We omit the lengthy table with which this section opens. It gives a list of basic and acid dye-stuffs with their surface tensions (detcrmined by a stalagmometer), and in parallel columns the average duration of life of fish in a 0.02 per cent solution and of tadpoles in a 0.1 per cent solution.-EDIToR.]

While the determination of the toxicities of ave-stuffs lies somewhat outside the scope of the present article we find it desirable to record the degree of toxicity with respect to both animals and plants of the various dye-stuffs examined. The results of the investigation of barley will appear shortly elsewhere; we give here only those respecting "Blitzfisch." Our investigations indicate that in basic dye-stuff solutions the surface activity is by no means a negligible factor. It is clearly connected with the complex magnitude which we term toxicity. The case is different with the acid dye-stuffs.

Two fishes each were place in 0.02 per cent solutions of pricric acid, Martius yellow, Bengal-rose, Brilliant-walk-blue, erythrosin, tetra-cyanol, Congo-red, dioinin-blue, Guinea green, fast-brown, Bordeaux, acid-violet, methyl-orange, naphtolyellow, acid-green, crystal poncean, quinolin-yellow, waterblue, and fast-red.

Picric acid and Martius-yellow proved very poisonous, killing the fish in 5 minutes. In fast-brown both were dead after 6 hours, and one was dead in brilliant walk-blue and in Bengal-rose each. Both were dea after 20 hours in tetracyanol and one each in crystal poncean and Congo-red. All the other fish were very lively.

Two fish each were then place in 0.1 per cent solution (i.e. five times as strong) of the following dye-stuffs ; cyanol, indigocarmine, Orange I, naphithol green, light green, heliotrope, eosin, phloxin, azo-blue, azo-ruby, anthra-quinon-green, brilliant Congo, and thio-carmine. After 2 hours the fish in
Orange I and in brilliant Congo were dead those in phloxin still exhibited reflex motons. We conclude therefore that nearly all the acid dye-stuffs are quite non-poisonous, including those with great surface activity. . .

\section{SUMMARY.}

1. Basic dye-stuffs in general exert a retarding action upon the rapidity of solution and an accelerating action on the rapidity of formation of a gelatine jelly. Hence they have a shrinking effect. As a usual thing the more colloidal the dye-stuff the greater the capacity for shrinking.

2. Acid dye-stuffs in many cases accelerate the rapidicy of solution and retar that of the formation of a gelatine jelly. Hence they have a swelling effect in many cases. However there are many also which have a shrinking effect (Bengal rose, erythrosin, azure blue, etc.).

3. Diffusion tests in gelatine show that in general, but by no means always, the capacity for and rapidity of diffusion of dye-stuffs run parallel to the permeability in plant tissues, but that we are not justified in regarding permeability as a simple process of jelly diffusion-the less so, since it has been shown that the capacity for and rapidity of dialysis through paper filters, etc., likewise usually run parallel to the capacity for and rapidity of jelly diffusion and permeability.

4. In general the greater or lesser capacity for jelly diffusion, and therewith the principle of ultra-filtration, may be regarded as a measure of the magnitude of the colloid particles, but this is by no means always so, since shrinking and swelling substances constantly alter the medium.

5. As in the alkaloids, so also in the dye-stuffs the dispersive power is primarily dependent upon the molecular weight. The capacity of substances to form colloidal aggregates is accordingly chiefly a function of the molecular weight.

6. The capacity of dye-stuffs to exert a shrinking or swelling effect is a factor which must be taken into account in the most various problems of biology as well as in the theory and technology of dyeing. Swelling favors permeability as a general thing, while shrinking favors storage. The problem of permeability requires consideration of this new factor for its solution.

7. New views with respect to the question of dyeing in general are based upon experiments in gelatine diffusion; among other things the effect of the addition of acids to acid dye-stuffs and of alkalies to basic dye-stuffs may be made clear. A dyeing process is in general colloidal in nature and not a chemical process governed by stoichiometric relations.

8. Measurements of the surface activity of dye-stuff solutions and determinations of the degree of toxicity show that in the case of basic dye-stuffs there is a definite (though not exclusive) connection between surface activity and toxicity with respect to taipoles and fish.

\section{NEW METHODS OF PREVENTING THE PROPAGATION OF TYPHUS}

A NEW "de-lousing" method has been developed by the Pasteur Institute of Paris and applied at its various centers throughout France. Clothing, bedding, etc., which are to be treated are hung $u p$ in a large oven or chamber, heated to 45 degrees $\mathrm{C}$. and containing 20 c.c. of chloropicrine per cubic meter of air (i.e. 1 to 50,000). Chloropicrine is well known as one of the asphyxiating gases used in the war. France possesses a considerable stock of this product, which like many others of its kind, can now be turned to a good advantage. Prof. G. Bertrand, of the Pasteur Institute, has recently examined the disinfectant and parasite-destroying properties of this substance. After a 20-minute treatment in this chamber, the parasites are entirely destroyed throughout the whole thickness of matresses and clothing, and the latter can be worn immediately afterwards without having suffered in the least. The present method, which is due to a careful research on the part of specialists, will render the greatest service against the propagation of typhus. 